\title{
Dezentralisierungsstrategien im Leistungsvergleich: Wirkungen von Dezentralisierungspolitik auf die Leistungsfähigkeit der Lokalsysteme in Deutschland, Frankreich und England
}

\section{Renate Reiter/Falk Ebinger/Stephan Grohs/Sabine Kuhlmann/Jörg Bogumil}

\section{Einleitung}

Die internationale Lokal- und Verwaltungsforschung geht heute weitgehend übereinstimmend von einem globalen Trend zur Dezentralisierung von Staatsaufgaben aus. ${ }^{1}$ Die Analyseperspektive bleibt dabei allerdings zumeist auf die Genese von Dezentralisierungspolitiken fokussiert. Welche Auswirkungen entsprechende Reformen indes auf die Performanz kommunalen Handelns haben ist bislang seltener untersucht worden. In diesem Artikel wenden wir uns genau diesen Effekten nationaler Dezentralisierungspolitiken auf die Leistungsfähigkeit öffentlicher Verwaltungen zu. Die Leitfrage nach den Wirkungen von Institutionenpolitik wurde im Rahmen eines Forschungsprojektes ${ }^{2}$ für Deutschland, Frankreich und Großbritannien jeweils am Beispiel je zweier Aufgabenfelder untersucht. Im Folgenden werden zunächst drei idealtypische Strategien der Aufgabendezentralisierung und die mit ihnen vermuteten Wirkungen erläutert. Anschließend stellen wir eine Heuristik zur Messung der Dezentralisierungswirkungen von Dezentralisierung auf die administrative Leistungsfähigkeit von Kommunen vor und präsentieren abschließend die zentralen Ergebnisse der empirischen Überprüfung.

\section{Dezentralisierungsstrategien und -wirkungen}

Dezentralisierungspolitik beruht auf der Annahme, dass die Form der staatlich-lokalen Aufgabenteilung im Hinblick auf die Performanz der öffentlichen Aufgabenerbringung einen Unterschied macht. Zunächst können drei Grundtypen oder -optionen der Dezentralisierung unterschieden und mit bestimmten theoretisch vermuteten Wirkungen in Verbindung gebracht werden: ${ }^{3}$

1 Vgl. Pollitt, Christopher/Bouckaert, Geert 2004: Public Management Reform: a Comparative Analysis, 2nd edition, Oxfort; Denters, Bas/Rose, Lawrence (Hrsg.) 2005: Comparing local governance: trends and developments, New York; Ahmad, Ehtisham/Brosio, Giorgio 2006: Handbook of Fiscal Federalism, Cheltenham.

2 Vgl. Kuhlmann, Sabine/Bogumil, Jörg/Ebinger, Falk/Grohs, Stephan/Reiter, Renate 2011: Dezentralisierung des Staates in Europa. Auswirkungen auf die kommunale Aufgabenerfüllung in Deutschland, Frankreich und Großbritannien, Wiesbaden.

3 Vgl. auch Benz, Arthur 2002: Die territoriale Dimension von Verwaltung, S. 209-214, in: König, Klaus (Hrsg.): Deutsche Verwaltung an der Wende zum 21. Jahrhundert. Baden-Baden, S. 207-228; Wollmann, Hellmut 2006: Staatsorganisation zwischen Territorial- und Funktionalprinzip im Ländervergleich - Varianten der Institutionalisierung auf der dezentral-lokalen Ebene, in: Bogumil, Jörg/Jann, Werner/Nullmeier, Frank (Hrsg.): Politik und Verwaltung. Sonderband 37 der Politischen Vierteljahresschrift. Wiesbaden, 424-452; Kuhlmann, Sabine 2010: Siegeszug der Terri- 
Die politische Dezentralisierung zielt auf eine vollständige Übertragung von Staatsaufgaben auf lokale Gebietskörperschaften. Im deutschen Kontext wird sie auch als „echte Kommunalisierung“ bezeichnet ${ }^{4}$. Dabei erhält ein lokal demokratisch gewähltes Vertretungsorgan die volle Zuständigkeit für die Entscheidung über die Planung, Finanzierung und Verwaltung der neuen Aufgabe. Diese geht also in den Bestand der Funktionen der kommunalen Selbstverwaltung über.

Die administrative Dezentralisierung stellt einen moderateren Typus der Neuordnung dar. In diesem Fall erhalten die gewählten Vertretungsorgane vor Ort keine autonomen Entscheidungs- und Kontrollkompetenzen. Zwar entscheiden die lokalen Behörden autonom über die konkrete Organisation der Aufgabenwahrnehmung, sie handeln jedoch als Agenten des Staates, d.h., sie bleiben nicht nur der staatlichen Rechts-, sondern auch Fachaufsicht unterworfen.

Die administrative Dekonzentration schließlich umfasst die Übertragung von zentralstaatlichen Aufgaben auf Behörden oder auch öffentlich-rechtliche Körperschaften, die auf einer subnationalen Verwaltungsebene angesiedelt, aber weiter Teil der staatlichen Verwaltungsorganisation im weiteren Sinne sind. Hierzu zählen zunächst dekonzentrierte (Fach-) Behörden des Staates ebenso wie neuere Behördenarten, insbesondere halbautonome, lokal angesiedelte staatliche Agenturen. ${ }^{5}$ Eine besondere Form der Dekonzentration, die vor allem im angelsächsischen Kontext anzutreffen ist, ist die horizontale Dekonzentration. Hier werden Aufgaben aus dem Funktionsportfolio der Kommunen herausgelöst und auf der gleichen Ebene in eigenständige Verwaltungsträger („Quangos“: Quasi-non-governmental organizations), die direkt dem Zentralstaat unterstellt sind, überführt. ${ }^{6}$

Der Kern der Diskussion um die Vor- und Nachteile der jeweiligen Dezentralisierungsform leitet sich von der geläufigen Unterscheidung zwischen einem Gebiets- ( $m u l$ ti purpose model) und einem Aufgabenorganisationsmodell (single purpose model) ab.? Während mit dem Gebietsorganisationsmodell eine horizontale, gebietsbezogene Ver-

torialität? Dezentralisierungsprofile und -wirkungen in Westeuropa, in: Schimanke, Dieter (Hrsg.): torialität? Dezentralisierungsprofile und -wirkungen in Westeuropa, in: Schimanke, Dieter (Hrsg.):
Verwaltung und Raum - Zur Diskussion um Leistungsfähigkeit und Integrationsfunktion von Verwaltungseinheiten. Baden-Baden, S. 101-126.

4 Wollmann, Hellmut 1997: „Echte Kommunalisierung“ der Verwaltungsaufgaben: innovatives Leitbild für umfassende Funktionalreform?, in: Landes- und Kommunalverwaltung (LKV) 7 (4), S. 105144; Burgi, Martin 2010: Kommunalisierung staatlicher Aufgaben - Möglichkeiten, Grenzen und Folgefragen aus rechtlicher Sicht, in: Bogumil, Jörg/Kuhlmann, Sabine (Hrsg.): Kommunale Aufgabenwahrnehmung im Wandel, 2010, S. 23 - 46.

5 Benz, Arthur 1996, Beyond the Public-Private Divide: Institutional Reform and Coopertive Policy Making, S.177ff. in: Benz, Arthur/Goetz, Klaus H. (Hrsg.): A new German public Sector?, Alershot etc., S. 165-188; Döhler, Marian 2007: Die politische Steuerung der Verwaltung. Eine empirische Studie über politisch-administrative Interaktion auf der Bundesebene. Baden-Baden.

6 Pollitt 2005 (Fn. 1); Skelcher, Chris 1998: The appointed state: Quasi-governmental organizations and democracy, Buckingham.

7 Vgl. Wagener, Frido 1979: Der öffentliche Dienst im Staat der Gegenwart, in: Veröffentlichungen der Vereinigung der Deutschen Staatsrechtslehrer, Heft 37, S. 215-266; Benz 2002 (Fn. 3); Wollmann, Hellmut 2006 (Fn. 3). waltungsorganisation gemeint ist, in der die Kommune als territoriale Einheit alle auf dieser Ebene anfallenden Aufgaben bündelt und in eigener politischer Verantwortlichkeit erfüllt, zielt das Aufgabenorganisationsmodell auf eine vertikale, funktionsbezogene Verwaltungsorganisation, in der für abgrenzbare Fachaufgaben jeweils ein spartenhaft ausgerichteter Behördenapparat von der (zentral)staatlichen bis auf die lokale Ebene existiert. In der vergleichenden Verwaltungswissenschaft werden diesen beiden idealtypischen Konfigurationen spezifische Auswirkungen auf die lokale Aufgabenerbringung und Performanz zugeschrieben. Demnach begünstigt das Gebietsorganisationsmodell die Koordination unterschiedlicher Fachpolitiken. Außerdem wird vermutet, dass die Möglichkeiten demokratischer Kontrolle vor Ort gestärkt werden, da ein breiteres Spektrum an Aufgaben lokalpolitisch unmittelbar von den Bürgern bzw. ihren Ratsvertretern kontrolliert und mitgestaltet werden kann. Als Nachteil wird postuliert, dass geringe Spielräume für die fachliche Spezialisierung der Akteure gegeben sind und so Effizienz- und Effektivitätsdefizite in Kauf genommen werden müssen. ${ }^{8}$ Die vermuteten Wirkungen des Aufgabenorganisationsmodells stellen sich entsprechend spiegelverkehrt dar. Übertragen auf die drei Dezentralisierungstypen kann angenommen werden, dass eine politische Dezentralisierung insbesondere die demokratische Kontrolle der Aufgabenwahrnehmung und die horizontale Koordination zwischen benachbarten Aufgaben stärkt, während Beziehungen zu anderen Verwaltungsebenen sowie die Leistungsfähigkeit und Einheitlichkeit des Vollzugs aufgrund schwindender Größeneffekte zurückgehen. Für die administrative Dezentralisierung kann im Vergleich zur politischen Dezentralisierung eine Abmilderung sowohl der positiven wie der negativen Effekte erwartet werden. Für die administrative Dekonzentration können schließlich die gegenteiligen Effekte der politischen Dezentralisierung erwartet werden.

Ein zentrales Problem der empirischen Bewertung institutioneller Politik besteht darin, dass die Leistungsfähigkeit öffentlicher Institutionen von zahlreichen intervenierenden Faktoren beeinflusst wird. Es ist daher oft nicht möglich, die Auswirkungen einer Reformmaßnahme klar zu isolieren. Zudem sind Ziele häufig unklar formuliert und einzelne Zieldimensionen widersprechen sich. Es ist also angebracht, einen mehrdimensionalen Rahmen zu entwickeln, der es ermöglicht, vielfältige auf Dezentralisierung gerichtete Perspektiven und Leistungserwartungen in sich aufzunehmen. Auf Basis der einschlägigen Literatur ${ }^{9}$ werden im Weiteren sechs Performanzdimensionen unterschieden, die ihrerseits den Aspekten der Input-Legitimation von Verwaltungshandeln (demokratische Kontrolle und Verantwortlichkeit, Transparenz), der Output-Legitimation sowie prozeduralen Gesichtspunkten (Koordinations- und Steuerungsfähigkeit, Effi-

$8 \quad$ Wagener 1979 (Fn. 7); Wollmann 1997 (Fn. 4); Wollmann 2006 (Fn. 3); Banner, Gerhard 2005 Local Government: A Strategic Resource in German Public Sector Reform, in: Hoffmann-Martinot, Vincent/Wollmann, Hellmut (Hrsg.): Comparing Public Sector Reforms in France and Germany, Wiesbaden.

9 Pollitt/Bouckaert 2004 (Fn. 1), S. 103 f.; Boyne, George A./Farrell, Catherine/Law, Jennifer/Powell, Martin/Walker, Richard M. 2003: Evaluating Public Management Reforms. Principles and Practice, Buckingham, S. $13 \mathrm{f}$ 
zienz und Effektivität) und schließlich dem Aspekt der Gleichheit (Equity) von Verwaltungsleistungen im inter-lokalen Vergleich Rechnung tragen. Zur Operationalisierung der Dimensionen wurden je mehrere Indikatoren definiert (vgl. Tabelle 1).

Die empirische Überprüfung dieses Analyserahmens erfolgte in den drei Untersuchungsländern Deutschland, Frankreich und England in je einem personenbezogenen Politikfeld (Sozialhilfepolitik: Frankreich, Eingliederungshilfe für Menschen mit Behinderung: Deutschland, Schulen: England) und einer technisch-planerischen Policy (Gewerbeaufsichts- und Immissionsschutzverwaltung: Deutschland, Stadt- und Raumplanung: Frankreich, England). Dazu wurden u.a. insgesamt 80 Interviews mit ausgewählten Experten jeweils auf kommunaler und Kreis- sowie regionaler Ebene und in übergeordneten staatlichen Instanzen durchgeführt. 10

Tabelle 1: Performanzdimensionen und -indikatoren.

\begin{tabular}{|c|c|}
\hline Performanz-Dimension & Indikatoren \\
\hline \multirow{5}{*}{$\begin{array}{l}\text { Demokratische Kontrolle \& } \\
\text { Verantwortlichkeit }\end{array}$} & Formale Entscheidungskompetenzen des Rates \\
\hline & Faktischer Gewinn an politischen Spielräumen \\
\hline & Beteiligung organisierter Interessen \\
\hline & Beteiligung von Bürgern \\
\hline & Transparenz von Entscheidungen \\
\hline \multirow{3}{*}{ Horizontale Koordination } & Koordinationsnotwendigkeit \\
\hline & Konfliktintensität der Abstimmungsprozesse \\
\hline & Beitrag zur Problemlösung \\
\hline \multirow{3}{*}{ Vertikale Koordination } & Koordinationsnotwendigkeit \\
\hline & Konfliktintensität der Abstimmungsprozesse \\
\hline & Beitrag zur Problemlösung \\
\hline \multirow{4}{*}{ Effizienz } & Ressourceneinsparung \\
\hline & Input-Output-Verhältnis \\
\hline & Skalenerträge \\
\hline & Verbunderträge \\
\hline \multirow{4}{*}{ Effektivität } & Legale Qualität \\
\hline & Professionelle Qualität \\
\hline & Kundenorientierung \\
\hline & Erreichbarkeit \\
\hline Homogenität & Überörtliche Gleichbehandlung \\
\hline
\end{tabular}

Quelle: Eigene Zusammenstellung.

\section{Empirische Auswirkungen von Dezentralisierung}

Deutschland, Frankreich und Großbritannien ${ }^{11}$ erscheinen als geeignete Beispiele zur Untersuchung der Performanzeffekte von Dezentralisierungspolitiken, da sie je unterschiedliche traditionelle Kommunalmodelle repräsentieren und zudem in den vergange-

10 Für Details vgl. Kuhlmann et al. 2011 (Fn. 2).

11 Im Weiteren beziehen wir uns allein auf England, da nach der Devolution die Entwicklung in Schottland, Wales und Nordirland teils unterschiedliche Entwicklungen zu beobachten waren, deren Erfassung den Rahmen des hier präsentierten Forschungsprojekts überstiegen hätte. nen zwei Dekaden Neuordnungen der zentral-lokalen Organisation der öffentlichen Aufgabenerbringung verfolgt haben. Deutschland und England können traditionell eher dem Gebietsorganisationsmodell zugerechnet werden, Frankreich dagegen stellt ein Beispiel für ein vom Aufgabenorganisationsmodell geprägtes Land dar. ${ }^{12}$ Hinsichtlich ihrer Reformstrategien wählten die Staaten unterschiedliche Wege. In Deutschland verfolgten die untersuchten Bundesländer (Baden-Württemberg und Niedersachsen) die Strategie der administrativen Dezentralisierung, Frankreich wählte überwiegend die Option der politischen Dezentralisierung und in England dominierte die Strategie der administrativen Dekonzentration. Ein Blick auf die Reformwirkungen in den insgesamt 15 Untersuchungsfällen bzw. zwölf Kreisen, Kommunen, Départements, Communes, Counties und Districts ergab hinsichtlich der einzelnen Performanzdimensionen zum Teil überraschende Ergebnisse.

\subsection{Demokratische Kontrolle und Verantwortlichkeit}

In Frankreich, wo die politische Dezentralisierung sowohl bei der Übertragung der Arbeitslosensozialhilfe (RMI) auf die Départements im Jahr 2004 als auch bei der Übertragung der Stadt- und Raumplanungsfunktion auf die Kommunen bereits im Jahr 1982 praktiziert worden war, konnte mit Blick auf die erste Performanzdimension zunächst eine formale Steigerung der politischen Verantwortung bei den lokalen Entscheidungsträgern konstatiert werden. Parallel dazu zeigte die Untersuchung, dass in den Gebietskörperschaften auch das politische Interesse an einer, erfolgreichen' Implementation der neuen Aufgabe (z.B. an der (Re-)Integration von RMI-Empfängern in den ersten Arbeitsmarkt) gestiegen ist. Dies ging allerdings kaum mit einer verstärkten Nutzer-, Bürger- oder Interessengruppenintegration in die politische Entscheidungsfindung einher. Dagegen kam es häufig zu einer Stärkung der lokalen oder departementalen Exekutive (des Bürgermeisters bzw. des Präsidenten des Generalrats). Lediglich in einzelnen Fällen konnten geringe Transparenzsteigerungen der lokalen Entscheidungsfindungsprozesse beobachtet werden. ${ }^{13}$

Auch in Baden-Württemberg waren nach der politischen Dezentralisierung der Eingliederungshilfe unerwartete Effekte zu beobachten: Die demokratischen Kontrolle und Verantwortlichkeit nahm nicht im erwarteten Umfang zu, da sich weder auf der Einzelfallebene noch bei der Steuerung des Gesamtsystems große Handlungsspielräume ergeben. Politisches Ermessen ist deshalb meist darauf begrenzt, finanziellen Druck an die Bürokratie weiterzureichen. Dennoch haben sich die Möglichkeiten Behinderter, ihre Interessen zu artikulieren, allein durch die räumliche Nähe und größere politische Sensibilität der Entscheidungsträger verbessert. Dessen ungeachtet ist die Transparenz des

12 Kuhlmann 2010 (Fn. 3).

13 Département 1, Direktor der Kommunalbehörde gegen soziale Ausgrenzung: Département 2, Leiter der Sozialintegrationsbehörde; vgl. auch: Thoenig, Jean-Claude 2005: Territorial Administration and Political Control: Decentralisation in France, S. 695f., in: Public Administration 83 (3), S. 685-708; Kuhlmann, Sabine 2009a: Politik- und Verwaltungsreform in Kontinentaleuropa: Subnationaler Institutionenwandel im deutsch-französischen Vergleich. Baden-Baden. S. 108. 
Meinungsfindungsprozesses niedrig, da professionelle Strukturen in den meisten Wahlkreisen die Repräsentation monopolisieren. Trotz der oft schmerzhaften Neugestaltung des Verhältnisses zwischen Kommunen auf der einen Seite und Verbänden auf der anderen Seite scheinen letztere gerade bei grundsätzlichen Fragen der Infrastruktur- und Angebotsentwicklung am stärksten von der politischen Dezentralisation zu profitieren, da der direkte Zugang zur Kommunalpolitik neue Gestaltungsmöglichkeiten eröffnet.

Mit der administrativen Dezentralisierung werden keine politischen Entscheidungskompetenzen auf die kommunale Ebene weitergegeben. Dennoch zeigen Analysen der Gewerbeaufsichtsverwaltung in Baden-Württemberg, dass Kommunalpolitiker sehr wohl beträchtlichen Einfluss auch auf untergeordnete Einzelverfahren nehmen können trotz hoher bürokratischer Professionalität, formell gebundener Entscheidungen und formal vorhandener staatlicher Aufsicht. Dies zeigt den permanenten Konflikt auf kommunaler Ebene, staatliche Regulierung durchzusetzen und gleichzeitig die Entwicklung lokaler Unternehmen zu fördern. Die Fachverwaltung sieht sich permanent mit politischen Forderungen konfrontiert, Auflagen und Kontrollen zu minimieren. „Demokratische Kontrolle“ erweist sich also hier als viel stärker ausgeprägt als in manchem Fall politischer Dezentralisierung.

Für die als administrative Dekonzentration bzw. horizontale Dekonzentration gefassten englischen Fälle bleibt die Beurteilung der demokratischen Qualität ambivalent. Im Feld der Schulen konnte durch die Einrichtung von Beiräten und Elternvertretungen auf der einen Seite eine verstärkte Partizipation (insbesondere der Eltern) beobachtet werden. ${ }^{14}$ Mit Einführung weitgehender Schulautonomie gewannen auch die Beiräte, in denen Eltern, Vertreter des councils und lokale Unternehmer repräsentiert sind, stärkeren Einfluss. In Verbindung mit der freien Schulwahl und nationalen Leistungsvergleichen zwischen Schulen kann von einer gestärkten „Konsumenten-Demokratie“ gesprochen werden. Auf der anderen Seite kann eine klare Schwächung demokratischer Kontrolle und Verantwortlichkeit hinsichtlich der Steuerungskapazitäten der gewählten councils konstatiert werden. Auch in der überlokalen Planung in England, bei der früher lokale Planungskompetenzen auf quasi-staatliche Planungskörperschaften übertragen wurden (eine Ausnahme bildet die politische Dezentralisierung im Bereich der Stadtplanung mit Einführung der neuen, auf der Distrikt-Ebene angesiedelten local developement frameworks LDF) muss eine Schwächung der demokratischen Kontrolle und Verantwortlichkeit beobachtet werden. Mit der Abschaffung der sog. "structure plans “ haben die counties ihren Einfluss weitgehend verloren. Die neue regionale Stufe mit der dreifachen Verwaltungsstruktur (regionale Planungskörperschaften, Regionalversammlungen und staatliche Government Offices) zeigt klare Transparenzdefizite und unklare demokratische Legitimation. Die Effekte der obligatorischen Bürgerbeteiligung bleiben aufgrund selektiver Partizipation (,übliche Verdächtige“) und moderater Einflüsse auf Entscheidungen schwach.

14 Vgl. auch Pollitt, Christopher/Birchall, Julie/Putman, Karen 1998: Decentralising Public Service Management. Houndmills.
Zusammenfassend kann die Annahme, wonach insbesondere politische Dezentralisierung eine Stärkung demokratischer Kontrolle und Verantwortlichkeit, vor Ort ‘ mit sich bringt, zwar bestätigt werden, allerdings wird die politische Dezentralisierung den Erwartungen bezüglich der Aufwertung demokratischer Kontrolle und Verantwortlichkeit nicht vollends gerecht. Die Effekte auf die direkte Partizipation von Bürgern und Betroffenen bleiben oftmals denkbar gering. Insgesamt kann keine der drei idealtypischen Dezentralisierungsstrategien als überlegen bezeichnet werden. Bei der politischen Dezentralisierung bleiben, ungeachtet eines erhöhten Maßes an formaler Legitimation der lokalen Politik nur geringe faktische Spielräume für mehr Bürgerbeteiligung; es sind vielmehr institutionelle Akteure und Interessenträger, die hier von der Aufwertung politischer Verantwortung profitieren. Bei der administrativen Dezentralisierung werden als unerwarteter Effekt - ebenfalls die politischen Akteure und Verantwortungsträger vor Ort gestärkt, das Ausmaß an formaler Legitimation und auch die Transparenz der Entscheidungsfindung bleiben allerdings schwach. Und die administrative Dekonzentration schließlich erhöht die Partizipationschancen der unterschiedlichen Stakeholder (betroffene Bürger, Interessengruppen), andere Aspekte der Legitimation und politischen Verantwortlichkeit kommen allerdings regelmäßig zu kurz.

\subsection{Horizontale Koordination}

Horizontale Koordination umfasst die Befähigung von Gebietskörperschaften, kohärente integrierte Lösungen für bestimmte öffentliche Probleme auf lokaler Ebene zu entwickeln. Hier ergaben sich für die Fälle zum Teil von der theoretischen Erwartung abweichende Ergebnisse. Am französischen Fall werden zugleich die Herausforderungen an die Fähigkeit der lokalen Politik und Verwaltung zur Komplexitätsbewältigung und auch die Bedeutung des lokal vorhandenen politischen und institutionellen Erbes in diesem Zusammenhang erkennbar. ${ }^{15}$ Beispielhaft kann man hierfür die Dezentralisierung des RMI herausgreifen. Da diese Sozialeingliederungspolitik in einzelnen Bereichen bereits vor ihrer vollständigen Dezentralisierung durch unterschiedliche Formen der staatlich-lokalen und der lokal öffentlich-privaten Arbeitsteilung gekennzeichnet war, wirkte sich die Aufgabenübertragung auf die Départements im Jahr 2004 nicht negativ auf die horizontale Koordinationsfähigkeit aus. Diese waren zum Zeitpunkt der Aufgabenübernahme bereits mit der Anforderung des umfassenden Komplexitätsmanagements vertraut 16 , konnten nunmehr allerdings ohne die ungeliebten staatlichen Koordinationspartner agieren, so dass die Dezentralisierung in der Summe eher positive Effekte. Die politische Dezentralisierung der Eingliederungshilfe in Baden-Württemberg übertrug ebenfalls komplexe horizontale Koordinationsfragen auf die kommunale Ebene. Nach beträchtlichen Anfangsschwierigkeiten und schweren Konflikten erlaubte die-

15 Kuhlmann 2009a (Fn. 16), S. 109.

16 IGAS (Inspection générale des affaires sociales) 2007 : Rapport sur la gestion du revenue minimum d'insertion (RMI). Présenté par J.-F. Chevallereaux, M. Laroque, M. Moleux, I. Rougier. Rapport RM2007-165P, Novembre 2007, http://lesrapports.ladocumentationfrancaise.fr/BRP/074000760/ 0000.pdf (am 05.01.2011). 
se Veränderung erstmals die Bündelung der meisten sozialen Dienstleistungen auf der lokalen Ebene. Die Zusammenfassung unter einem Dach ermöglichte die Glättung der Schnittstellen mit Sozialamt, Gesundheitsamt, Jugendhilfe etc. Als wesentlicher Vorteil der Dezentralisierung erwies sich die Möglichkeit, die Anwendung des Fallmanagements auszuweiten. Mit der höheren örtlichen Nähe und der Kontinuität der Beziehungen wuchs auch das Wissen der Fallmanager über die lokalen Dienstleistungsanbieter, was Steuerungsoptionen eröffnete und sich positiv auf die Qualität auswirkte. Dieser bemerkenswerten Entwicklung der horizontalen Koordination innerhalb vieler Kommunen stehen beträchtliche Nachteile in der horizontalen Koordination zwischen den lokalen Einheiten gegenüber. Die Abstimmung der Positionen und Vorgehensweisen zwischen den Landkreisen erfordert umfangreiche und aufwändige interkommunale Verhandlungen. In der Summe überwiegen die Vorteile der Dezentralisierung die Nachteile.

Im Fall der administrativ dezentralisierten Gewerbeaufsicht in Baden-Württemberg sind die Urteile über die inneradministrative horizontale Koordination gemischt. Während in manchen Kreisen die Erleichterung der täglichen Koordination gelobt wurde, bemerkten andere keine Veränderung: Alle Experten betonten zwar das Potenzial für eine weitere Optimierung der Koordinierungsprozesse, innerbehördliche Kämpfe, verwurzelte Routinen und gegenläufige politische Interessen ließen die Realisierung jedoch sehr unwahrscheinlich erscheinen. Gleichzeitig erwies sich die Entwicklung der horizontalen Koordination und Kommunikation zwischen den 44 Stadt- und Landkreisen im Vergleich zu den Binnenbeziehungen als sehr viel wichtiger: Das Politikfeld erfordert einerseits eine Harmonisierung des Verwaltungshandelns, andererseits kann den Kapazitätsverlusten nur durch intensive Kooperation und die Weitergabe von Know-how begegnet werden. Informelle Netzwerke sind die Basis für die meisten dieser Austauschbeziehungen, diese sind jedoch an persönliche Kontakte gebunden und erodieren über die Zeit. Kreise mit geringen Kapazitäten haben bereits wenig Jahre nach der Dezentralisierung keinen Zugang mehr zu dieser Ressource.

Für den englischen Fall administrativer Dekonzentration im Schulbereich zeigen sich klare Defizite in der horizontalen Koordination. Aufgrund der abnehmenden Planungskapazität der Local Education Authority (LEA) konkurrieren die Schulen nun um Personal und die Nutzung öffentlicher Angebote wie z.B. Räume oder Sportanlagen anstatt hier zu kooperieren. Regelmäßig wurde auch von einer Schwächung der Koordination zwischen Schulen und lokalen Behörden in Schnittstellenbereichen wie Jugendhilfe, Kriminalprävention, etc. berichtet. Diese negativen Folgen zogen Reformen mit dem Ziel einer besseren Koordination mit anderen Verwaltungsbereichen nach sich (Integration der Bildungsverwaltung mit der Kinder- und Jugendhilfe etc.). Im Gegenzug wurde die vertikale Koordination durch nationale Curricula, Leistungsvergleiche sowie staatliche Inspektorate (OFSTED) gestärkt. Auf der Regionalplanungsebene in England finden wir typische Effekte einer Single-purpose-Verwaltung. Hier sind die Planungsprozesse entkoppelt von anderen Politikfeldern, da sich die monofunktionalen Planungskörperschaften auf die Planungsverantwortung allein konzentrieren. Die Wahrnehmung anderer signifikanter regionaler Aufgaben - regionale Wirtschafts- und Strukturent- wicklung - ist anderen Körperschaften zugewiesen, was einen bestimmten Grad der institutionellen Rivalität schafft.

Insgesamt zeigt der Vergleich der drei Dezentralisierungsstrategien mit Blick auf die horizontale Koordination widersprüchliche Ergebnisse. Während für die Fälle der politischen Dezentralisierung mehrheitlich positive oder zumindest neutrale Entwicklungen beobachtet werden konnten, schuf die administrative Dezentralisierung im Bereich des Immissionsschutzes und der Gewerbeaufsicht einen massiven Bedarf an horizontaler Koordination zwischen lokalen Einheiten. Die administrative Dekonzentration in England bringt offensichtliche Koordinationsdefizite hervor. Offensichtlich variieren aber Koordinationsnotwendigkeiten zwischen unterschiedlichen Leistungsarten (personenbezogen sozial; technisch-planerisch) stark.

\subsection{Vertikale Koordination}

Im französischen Fall müssen Defizite in der vertikalen Koordination zwischen den Gebietskörperschaften und dem Staat diagnostiziert werden. Die Koordination zwischen den Ebenen wurde sowohl im Bereich der RMI-Sozialhilfe als auch im Feld der Stadtund Raumplanung nach dem Aufgabentransfer auf ein Minimum der gesetzlich vorgeschriebenen intergouvernementalen Abstimmung reduziert (dies betraf z. B. den Informationsaustausch in Angelegenheiten des Zivilschutzes im Planungsbereich). Der Abbau der staatlich-lokalen Koordinationsbeziehungen war im Falle der RMIEingliederungspolitik eher nachteilig für die Handlungsfähigkeit der betroffenen Départements, denn der Gesetzgeber flankierte die Aufgabeübertragung nicht mit der Übertragung auch der administrativen Weisungskompetenzen von den vormaligen staatlichen Aufgabenträgern auf die neuen Träger, die Generalräte. Im Planungsbereich hingegen funktioniert die vertikale Koordination im Anschluss an die Aufgabenübertragung gut. Neben den Kommunen sind die anderen Gebietskörperschaften hier allenfalls in einzelnen Funktionsbereichen an der Aufgabenerbringung beteiligt, so dass die mögliche ,Reibungsfläche“ hier von vorn herein klein ist. Außerdem sind die Beziehungen zwischen den Ebenen im Feld der Planung stark von fachlich orientierten Interaktionen geprägt; hier mindert die Existenz von Ebenen-übergreifenden Fachbruderschaften häufig das Risiko der nachlassenden vertikalen Koordinationsfähigkeit.

Im baden-württembergischen Fall der Eingliederungshilfe ist die vertikale Koordination fast exklusiv auf die Beziehung zwischen den kommunalen Einheiten und dem Kommunalverband Jugend und Soziales (KVJS) als gemeinsame Einrichtung der Kommunen beschränkt. Entmachtet und verschlankt agiert diese Nachfolgeorganisation der ehemaligen Landeswohlfahrtsverbände vorrangig als Serviceeinrichtung, z.B. bei der fachlichen Beurteilung der Bedürfnisse behinderter Personen. In Ermangelung hierarchischer Kompetenzen und aufgrund der Abneigung lokaler Entscheidungsträger, sich einheitlichen Standards zu unterwerfen, wird die Rolle des KVJS mehr und mehr marginalisiert und die vertikale Koordination weiter geschwächt. Die administrative Dezentralisierung der Gewerbeaufsicht in Baden-Württemberg machte spezialisiertes Wissen zu einem knappen Gut. Der vertikale Austausch zwischen staatlicher und kommunaler Ebene könnte zur Behebung dieses Engpasses beitragen. Dies ist jedoch nur 
sehr begrenzt der Fall: Nicht nur verfügen die Regierungspräsidien häufig nicht mehr über die Kapazitäten für die fachliche Unterstützung der Kommunen, sie gaben soweit möglich auch die Rolle einer aktiven Aufsichtsebene auf und wechselten wann immer möglich in die Rolle eines Beraters, der jedoch kaum den Unterstützungsbedarf der Kommunen befriedigen kann.

Die englische administrative Dekonzentration im Schulbereich ist von einer zunehmenden vertikalen Integration gekennzeichnet. Die Zahl der Schnittstellen zwischen Repräsentanten des Staates und den einzelnen Schulen wie auch den lokalen Behörden hat sich vergrößert. In beiden Fällen hat sich der autoritäre Interventionsstil der staatlichen Verwaltung verstärkt und manifestiert sich speziell in einem starren System von Inspektionen und Performance-Management. Im Feld der Planung fand eine klare Differenzierung der Zuständigkeiten in der vertikalen Dimension statt. Trotz der gewachsenen Autonomie blieb die Ebene der districts Gegenstand einer Zahl von vertikalen Interventionen. Auf der einen Seite haben sich die oft beobachteten direkten ErmessensInterventionen des Secretary of State verringert. Auf der anderen Seite wird die Entwicklung der Stadtplanung von den Government Offices beaufsichtigt und ist Gegenstand einer Überprüfung der staatlichen Planungsinspektorate. Konflikte entwickeln sich speziell im Falle von Diskrepanzen zwischen lokaler und regionaler Planung, oft verstärkt durch unterschiedliche politische Präferenzen.

Fasst man die empirischen Beobachtungen zusammen, führte die Dezentralisierung insgesamt zu einem Anstieg der vertikalen Koordinationsbedarfe. Die tatsächliche Koordinationsfähigkeit hielt mit dieser Entwicklung in den meisten untersuchten Fällen allerdings nicht Schritt. So traten in einigen Fällen der politischen Dezentralisierung aufgrund unklarer Weisungsregelungen zwischen den unterschiedlichen lokalen Gebietskörperschaften Streitigkeiten über die Kompetenzen der gebietskörperschaftlichen Akteure bei der Aufgabenerbringung auf und in den britischen Fällen der horizontalen Dezentralisierung kam es überwiegend zur Etablierung neuer hierarchischer top-downSteuerung durch den Staat. Zwischen diesen Polen erweisen sich die politische Dezentralisierung in der französischen Stadt- und Rauplanung und die administrative Dezentralisierung von Immissionsschutz und Gewerbeaufsicht als Ausnahmen; sie stehen beispielhaft für ausgewogen strukturierte Beziehungen nach der Dezentralisierung.

\subsection{Effizienz}

Im Falle Frankreichs war die politische Dezentralisierung insbesondere der Sozialhilfe RMI mit Effizienzeinbußen verbunden. Diese rührten allerdings weniger aus der Aufgabenübertragung her, als vielmehr zum einen daraus, dass der Zentralstaat den Départements zusammen mit der Verantwortung für den RMI ein Reihe von aufgabenbezogenen Risiken übertrug. $\mathrm{Zu}$ nennen sind hier das Risiko der Rückholung unrechtmäßig ausgezahlter Leistungen an RMI-Empfänger ${ }^{17}$ und auch das Risiko des Ausgleichs ar-

17 Im Jahr 2008 summierte sich dies auf etwa 120 Millionen Euro oder knapp ein Prozent der RMIGesamtausgaben der Départements (ODAS, Observatoire national de l'action sociale décentralisée beitsmarkt- und konjunkturbedingt schwankender Kosten dieser Arbeitslosensozialhilfe. ${ }^{18}$ Zum anderen hingen Kostensteigerungen mit dem erklärten Willen der meisten Generalräte zusammen, nach der vollständigen Aufgabenübernahme eine konstant hohe Leistungsqualität auch im Angesicht eines vom Gesetzgeber veränderten und erweiterten Leistungsrechts aufrecht zu erhalten. Um diesem Anspruch gerecht werden zu können, wurden in den meisten Départements Investitionen in zusätzliches Personal sowie in die Anpassung der internen Managementprozesse und technischen Unterstützungssysteme der Leistungserbringung erforderlich ${ }^{19}$. Zusammengefasst deuten die empirischen Beobachtungen auf einen kurzfristigen Anstieg der Kosten ${ }^{20}$. Dies trifft nicht zu auf den zweiten Fall der politischen Dezentralisierung in Frankreich, die Stadt- und Raumplanungspolitik. Hier haben sich die anfänglichen Transaktionskosten zwei Jahrzehnte nach der Aufgabenübertragung amortisiert, so dass weder auf Seiten der größeren Städte noch auf Seiten der kleineren Gemeinden Effizienzeffekte abgeschätzt werden können.

Im Fall der politischen Dezentralisierung der Eingliederungshilfe nahm die Kosteneffizienz stark ab. Dies hängt sowohl mit der Fragmentierung der Aufgaben und dem Verlust zentral bereitgestellter Aufgaben, als auch mit den Investitionen in den Aufbau von Fall-Management-Kapazitäten zusammen. Gleichzeitig stieg der politische Druck zur Kostensenkung. Aufgrund der ungleichen Verteilung von qualifiziertem Personal boten sich den Verwaltungen in den Gebietskörperschaften sehr unterschiedliche Startbedingungen verteilt. ${ }^{21}$ Mittlerweile haben so gut wie alle Kommunen akzeptiert, dass die Investition in Fall-Management Kapazitäten der einzige Weg ist, die absehbare Kostenexplosion zu kontrollieren. Eine bessere Koordination zwischen den lokalen Dienstleistungen führt bereits heute zu Effizienzgewinnen. Zusammenfassend kann behauptet werden, dass erst die Dezentralisierung die von Fachleuten als unumgänglich angesehenen Investitionen in individuelle Betreuungslösungen politisch möglich machte.

Der Fall der Gewerbeaufsicht in Baden-Württemberg kann als typisches Beispiel für eine politisch motivierte administrative Dezentralisierung gesehen werden. Deshalb nahmen die Kommunen - begierig, zusätzliche Vollzugskompetenzen zu übernehmen eine zwanzigprozentige Kürzung der staatlichen Kostendeckung hin. Diese Einsparung wurde oft durch die Übernahme einer geringeren als den Kommunen rechnerisch zuste-

2009: Dépenses départementales d'action sociale en 2008: la fin d'un cycle. La lettre de l'Odas, S. 9).

18 Clerc, Denis 2009: Décryptage de la loi du 1er décembre 2008 (2e partie): Les mesures relatives au revenu de solidarité active, La lettre de l'insertion $\mathrm{n}^{\circ} 9$ (Avril 2009).

19 Kuhlmann 2009a (Fn. 16); Kuhlmann, Sabine 2009b: Frankreich: Wandlungen des jakobinischen Staates - "faire mieux avec moins"?, in: Europäisches Zentrum für Föderalismus-Forschung Tübingen (Hrsg.): Jahrbuch des Föderalismus 2009, Baden-Baden, S. 263-274.

20 Interview Département 1, Direktor der Départements-Behörde gegen soziale Ausgrenzung; Interview Département 2, Leiter der Sozialintegrationsbehörde.

21 Vgl. Ebinger, Falk/Bogumil, Jörg 2008: Grenzen der Subsidiarität - Verwaltungsreform und Kommunalisierung in den Ländern, in: Heinelt, Huber/Vetter, Angelika (Hrsg.): Lokale Politikforschung heute, Wiesbaden, S. 165-196. 
henden Zahl von Fachmitarbeitern erfüllt. Während große Verwaltungen dies noch verkraften können, sehen sich kleinere Kreise mit anhaltenden strukturellen Problemen konfrontiert. Folgt man der generellen Einschätzung der Interviewpartner, überschreiten Kompetenzdefizite und daraus resultierende Folgekosten für Verwaltung, Wirtschaft und Bürger bei weitem die unmittelbaren Einsparungen der Dezentralisierung.

Im englischen Schulbereich bleiben die Effizienzzuwächse bis zum heutigen Tag umstritten. Die meisten Zuwächse können nicht den administrativen Veränderungen, sondern den neuen flexiblen Budgets zugeschrieben werden, die auch unabhängig von der Dekonzentration denkbar gewesen wären. ${ }^{22}$ Allerdings gibt es Anzeichen für höhere Verwaltungskosten durch die Internalisierung und De-Professionalisierung von administrativen Aufgaben (z.B. Personal, Gebäudeunterhaltung). Im Feld der Planung ist nur eine vorläufige Beurteilung der Effizienzgewinne möglich. Mit der Abschaffung von „structure plans“ auf der County-Ebene wurde das Personal erheblich reduziert. Die neuen regionalen Planungskörperschaften sind schlanker, was auf den ersten Blick Effizienzgewinne nahe legt. Allerdings ist auch mehr Personal in den staatlichen Regierungsbüros und den Planungsinspektoraten gebunden, sodass insgesamt Effizienzgewinne unsicher sind.

Während der Vergleich der Effekte der Dezentralisierungsstrategien auf die Effizienz zu unklaren Ergebnissen führt, kann ein politikfeldbezogener Effekt beobachtet werden: hauptsächlich technische Aufgaben liefern nicht in jedem Fall Gewinne, während in beiden Fällen der Integration von Dienstleistungen langfristig positive Effekte erwartet werden.

\subsection{Effektivität}

Im Fall des französischen RMI war es schwer, die Effekte der Dezentralisierung eindeutig zu identifizieren, denn der Gesetzgeber verband die Aufgabenübertragung hier mit einer inhaltlichen politikfeldbezogenen Reform, die die Départements vor die Herausforderung organisatorischer und personeller Umstellungen stellte. Um die neuen gesetzlichen Standards zur Eingliederung zu erreichen, investierten viele Départements in eine Professionalisierung ihres Personals, die allerdings bis zum Abschluss der empirischen Untersuchung noch keine Früchte hervorgebracht hatte. ${ }^{23}$ Außerdem brachte die neue Orientierung der RMI-Politik in Richtung einer aktivierenden Arbeitsmarktpolitik die Notwendigkeit mit sich, bereits existierende und oft gut funktionierende Partnerschaften neu zu formieren, was zu wenigstens kurzzeitigen Verlusten in der Effektivität hinsichtlich der fachlichen Qualität beitrug. ${ }^{24}$ Alles in allem nahm so die Effektivität in der Folge der Dezentralisation zwar nicht ab, konnte aber aufgrund der Dualität von Dezentralisierung und Politikreformen auch nicht verbessert werden; Verbesserungen er-

22 vgl. Levacic, Rosalind 1994: Evaluating the Performance of Quasi-Markets in Education, in: Bartlett, Will/Propper, Carol/Wilson, David/LeGrand, Julien (Hrsg.): Quasi-Markets in the Welfare lett, Will/Proppe

23 Interview Département 1/Stadt 1, Leiterin kommunales Sozialzentrum.

24 Interview Département 1, Direktor der Behörde gegen soziale Ausgrenzung; IGAS 2007 (Fn. 20). scheinen allenfalls in der Zukunft möglich. Im Falle der zeitlich weiter zurückliegenden politischen Dezentralisierung der Stadt- und Raumplanung verlief die Entwicklung entsprechend. Hier trug die Professionalisierung der lokalen Verwaltung in großen und mittleren Städten bereits während der 1980er Jahre ${ }^{25}$ mittelfristig dazu bei, einen hohen fachlichen Standard nicht nur aufrechtzuerhalten, sondern diesen etwa im Hinblick auf die Kundennähe der Verwaltung noch zu verbessern. ${ }^{26}$

Im Fall der baden-württembergischen Eingliederungshilfe wird die Bedeutung politischer Unterstützung für einen effektiven Aufgabenvollzug offensichtlich. Vorteilhaft institutionalisierte Einheiten konnten umfassende Fallmanagementkapazitäten etablieren und sowohl die Binnen- als auch die Außenbeziehungen in den Kommunen optimieren. Örtliche Nähe und die konstruktive Zusammenarbeit mit den Leistungsanbietern ermöglichte in diesen Fällen eine Verbesserung der Angebotsstruktur und der Leistungsqualität. In anderen, weniger vorteilhaft institutionalisierten Fällen, berichten Experten und Beteiligte von hohem politischem Druck auf die Verwaltung, insbesondere die Fallmanager, sowohl Kosten einzusparen oder aber den Wünschen der etablierten örtlichen Anbieter entgegen zu kommen. So erscheint das Ergebnis der Dezentralisierung hier stark vom Willen der politischen Führung der Gebietskörperschaften abzuhängen, eine leistungsfähige Verwaltung zu etablieren und diese auch politisch zu unterstützen.

Für die administrative Dezentralisierung der Gewerbeaufsicht muss eindeutig ein negatives Fazit gezogen werden: Beträchtliche Defizite resultieren aus dem starken Verlust von Fachwissen und dem Unterschreiten kritischer Größen für einen effektiven Verwaltungsvollzug. Anstelle von etwa 50 bis 70 Experten in den ehemals neun staatlichen Immissionsschutz- und Gewerbeaufsichtsbehörden, beschäftigen die 44 kommunalen Einheiten jeweils nur zwischen 3 und 15 Fachmitarbeiter. Diese sind nun häufig für bis zu zehn Branchen verantwortlich - eine Kompetenzspanne, in der kaum das notwendige profunde Wissen vorgehalten werden kann. Interviewpartner berichteten von gestiegenen Bearbeitungszeiten oder fachlicher Verflachung der Arbeit, abnehmender Servicequalität und einem Reputationsverlust bei den Kunden der Verwaltung.

Im englischen Fall der administrativen Konzentration im Schulbereich hat entgegen der Reformziele keine klare Standardisierung der Ergebnisse gemessen an Lernstandards und erreichten Abschlüssen stattgefunden. Unterschiede zwischen den Schulen können weiterhin eher auf den sozialen Hintergrund der Schüler und des Schulumfelds zurückgeführt werden, denn auf die Leistungsfähigkeit der Schule. ${ }^{27}$ Zudem führt die Autonomisierung der Schulen zu schlechteren Koordinationsleistungen was sich insbesondere in mangelhafter Integration von Bildung und Sozialarbeit und anderen Aufgabenfeldern zeigt. Zusammengefasst gibt es nur kleine Anzeichen für ein Anwachsen der Effektivität, die der Dekonzentration im Bildungssektor zugeschrieben werden können.

25 Vgl. Kuhlmann, Sabine 2008: Dezentralisierung in Frankreich: Ende der unteilbaren Republik?, in: der moderne staat - dms, 1(1), S. 201-220.

26 Gemeinde 2, Leiter des städtischen Bauamtes.

27 Interview: County 1, Leiter der Local Education Authority. 
Die Effektivität der dekonzentrierten Ebene der Regionalplanung wird von politischen und juristischen Hindernissen, sowie Widerständen der lokale Ebene, die die Übernahme und eine effektive Umsetzung der regionalen Raumstrategien blockiert, gehemmt, so dass hier fünf Jahre nach der Reform immer noch ein weitgehender NichtVollzug zu vermelden war.

Vergleicht man die drei Dezentralisierungsformen, scheint die politische Dezentralisierung die gegenüber den beiden anderen Formen überlegene Option zu sein. Insbesondere im Bereich der technisch-planerischen Aufgabenarten zeigten sich hierbei vergleichsweise ausgeprägte positive Effekte in Form von Effektivitätsverbesserungen. Insgesamt tragen auch hier erneut kontextuelle Faktoren wie die Art der Policy, die Dauer der Reformwirkung oder die Gemeinde- oder Kreisgröße Wesentliches zur Erklärung der beobachteten Performanzänderungen im Anschluss an den Aufgabentransfer bei.

\subsection{Heterogenität der Leistungsproduktion im inter-lokalen Vergleich}

Die politische Dezentralisierung des RMI in Frankreich trug klar zu einem Anwachsen in der tatsächlich existierenden transterritorialen Heterogenität bei der Produktion sozialer Dienstleistungen bei. ${ }^{28}$ Départments mit restriktiver Haushaltssituation waren im Gegensatz zu finanziell gesunden Départements selten in der Lage, ihr Angebot an Integrationsdienstleistungen zugunsten der RMI-Empfänger in einer Weise, die für die Implementation einer tatsächlich aktivierenden Arbeitsmarkt- und Eingliederungspolitik notwendig gewesen wäre, auszubauen. ${ }^{29}$ Im Fall der Stadt- und Raumplanungspolitik war eine Heterogenisierung der Leistungen nach der Dezentralisierung - soweit dies die fachliche und rechtliche Qualität der Aufgabenerbringung betrifft - nicht feststellbar.

In Baden-Württemberg führten die unterschiedlichen Startbedingungen und Managementphilosophien im Bereich der Eingliederungshilfe zu sehr verschiedenen Institutionalisierungen und Arbeitsansätzen. Während einige Stadt- und Landkreise integrierte Sozialabteilungen installierten oder in den Aufbau von Fallmanagement-Kapazitäten investierten, behandelten andere diese neue Zuständigkeit eher stiefmütterlich. Und während die Mehrheit der Landkreise versucht, eine allgemein akzeptierte Balance zwischen Serviceniveau und Kosteneinsparungen zu finden, beschränkten sich einige auf Maßnahmen zur kurzfristigen Kostensenkung. Harmonisierungsversuche erwiesen sich als konfliktträchtig und zeitaufwändig, ohne das die politisch bedingten Unterschiede zwischen den Stadt- und Landkreisen im Kern angeglichen werden konnten. Die administrative Dezentralisierung der Immissionsschutz- und Gewerbeaufsichtsverwaltung in Baden-Württemberg führte zu einer beträchtlichen Heterogenität zwischen den Gebietskörperschaften. Ungleiche politische Unterstützung, Ressourcenverteilung, Zugang zu

28 Vgl. Mission parlementaire sur les droits connexes dans le cadre de la généralisation du RSA 2009: Rapport de Madame Sylvie Desmarescaux, Sénateur. C. Hesse, Membre de l'inspection générale des affaires sociales, Mai 2009, http://lesrapports.ladocumentationfrancaise.fr/BRP/094000221/0000. $\operatorname{pdf}(05.01 .2011)$, S. 13.

29 Interview Département 2, Leiter der Sozialintegrationsbehörde.
Fortbildungen wie auch zu den informellen Netzwerken führen zu einem weiteren Auseinanderlaufen der Leistungen.

Infolge der horizontalen Dezentralisierung stieg in England die Heterogenität sowohl zwischen einzelnen Schulen als auch zwischen den Kommunen an. Einige Kommentatoren sehen dies als eine intendierte Konsequenz aus dem Anspruch wettbewerbsorientierten Reform. Trotz dieser wachsenden Diskrepanzen gibt es aber einen leichten Trend zu durchschnittlich besserer Leistung durch Wettbewerb. Auf der anderen Seite wird die vorher bestehende Ausgangslage einzelner Schulen bezüglich des sozialen Hintergrundes der Schüler, der Gebäudeunterhaltung und der Rekrutierung der Lehrer weiter verstärkt (,Wer hat, dem wird gegeben“). In beiden counties kamen diese Ungleichheiten auf und wurden als ein Hauptproblem für den Aufbau county-weiter Strategien zur Verbesserung der Bildungspolitik bezeichnet. Im Gebiet der englischen Planung sind durch die Rolle der Planungsinspektorate und des Secretary of State sowie der Standardisierung der Regierungsbüros konvergente Entwicklungen bemerkbar. Beträchtliche Varianz in den Planungsentwürfen ist die Folge von verschiedenen nationalen Planungszielen für die Regionen, die sich auf Bauland für erschwingliche Eigenheime im Süden, die Bewältigung des Strukturwandels im Norden berufen.

Zusammengefasst können ähnliche Ergebnisse für alle drei Dezentralisierungsoptionen beobachtet werden: Die Heterogenität nahm in allen Fällen zu, wobei die Hauptursachen dieser Entwicklung der Mangel an eigenen finanziellen Ressourcen der lokalen Gebietskörperschaften sowie fehlender politischer Wille zur interlokal abgestimmten Homogenisierung der Leistungserbringung waren.

\section{Schlussfolgerung}

In diesem Beitrag wurden unterschiedliche Dezentralisierungsstrategien - die politische und die administrative Dezentralisierung sowie die administrative Dekonzentration - in ihrer Eigenschaft als institutionelle Reformpolitiken auf ihre Auswirkungen auf die administrative Leistungsfähigkeit („Performanz“) von Kommunen und Kreisen in unterschiedlichen Politikbereichen überprüft. Mit Hilfe eines Analyserahmens zur vergleichenden Analyse von Dezentralisierungseffekten wurden insgesamt 16 Fallstudien in den drei Ländern durchgeführt. Ein zentrales Ergebnis ist die Erkenntnis, dass sich mehrere der bisherigen theoretischen Annahmen über die Wirkungen von Dezentralisierungspolitiken nicht bestätigt haben. Ein weiteres Ergebnis liegt darin, dass ein direkter Zusammenhang zwischen der gewählten Dezentralisierungsstrategie und den beobachteten Leistungseffekten nicht nachgewiesen werden konnte. So vergrößert z.B. politische Dezentralisierung die Möglichkeiten der Politik sowie der Bürger ,vor Ort' zur demokratischen Kontrolle zunächst nur formal. Eine Steigerung der politischen Verantwortlichkeit von Verwaltungen ist nicht notwendig mit dieser Strategie verbunden. Administrative Dezentralisierung und administrative Dekonzentration wiederum sind nicht notwendig mit einer Steigerung der Effizienz und/oder Effektivität des Verwaltungshandelns verbunden. Die Erklärungskraft institutionalistischer Annahmen, die auch in der Realität mitunter als Begründung für dezentralisierungsbewährte Verwal- 
tungsreformpolitiken herangezogen werden - dies ist in unserer Studie sichtbar geworden - ist offenkundig begrenzt. Hingegen deuteten sich stärkere Zusammenhänge zwischen der Performanz der Gebietskörperschaften einerseits und der Art des von Dezentralisierung betroffenen Politikfelds (technisch-planerisch oder personenbezogen), dem Spektrum an politischen Interessen innerhalb der Gebietskörperschaft und der seit der jeweiligen Reform verstrichenen Zeit an. Insgesamt lautet die Schlussfolgerung daher: Dezentralisierung spielt als institutionelle Modernisierungspolitik eine Rolle, die Richtung des Wandels bestimmter Indikatoren von Verwaltungsperformanz ist allerdings nicht institutionell determiniert und kann daher nicht ex-ante aus der je gewählten Dezentralisierungsstrategie abgeleitet werden. Daher empfiehlt sich bei der Untersuchung der Effekte von Dezentralisierungspolitiken die Wahl eines integrativen, nichtinstitutionelle Kontextfaktoren mitberücksichtigenden Forschungsdesigns. 